\title{
Two-Dimensional Ising Spin Glasses with Nonzero Ordering Temperatures
}

\author{
N. Lemke*,† and I. A. Campbell ${ }^{\ddagger}$ \\ Laboratoire de Physique des Solides, Université Paris-Sud, Bâtiment 510, Centre Universitaire, 91405 Orsay Cédex, France
}

(Received 19 March 1996)

\begin{abstract}
We demonstrate numerically that, for Ising spins on square lattices with ferromagnetic second neighbor interactions and random near neighbor interactions, two-dimensional Ising spin glass order with a nonzero freezing temperature can occur. We compare some of the physical properties of these spin glasses with those of standard spin glasses in higher dimensions. [S0031-9007(96)00432-2]

PACS numbers: 75.50.Lk, 64.60.Cn
\end{abstract}

In dimension two, an Ising spin glass (ISG) with random near neighbor interactions does not order above zero temperature [1,2]. Implicitly, this has been taken to mean that two-dimensional (2D) ISGs with ordering at finite temperatures do not exist. Here we show that this assumption is unfounded by exhibiting a family of 2D Ising systems with a different set of interactions, which show spin glass ordering at finite temperatures. We find that the physical properties of these 2D ISG systems resemble closely those of standard ISGs in higher dimensions.

Consider a square lattice of Ising spins with second nearest neighbor ferromagnetic interactions of strength $J$. This clearly consists of two interpenetrating but noninteracting square sublattices which will each order ferromagnetically at the Onsager Curie temperature $T_{c}=$ $2.27 \mathrm{~J}$. Now introduce random near neighbor interactions of strength $\pm \lambda J$. At low temperatures when the spins are frozen, each sublattice will exert effective random fields on the spins of the other sublattice. It is well established [3] that a 2D Ising ferromagnet breaks up into finite size domains under the influence of a random field, however small. At low temperatures each sublattice of the present system will then be ordered in randomly arranged ferromagnetic domains of finite size. However, in contrast to the true 2D random field Ising system, here the "effective random fields" will average to zero at high temperatures, so we can expect a critical temperature $T_{g}$ separating a high temperature paramagnetic regime [with the autocorrelation function $q(t)$ relaxing to zero at long time $t$ ] from a low temperature regime where $q(t)$ does not go to zero, corresponding to ordering with a nonzero Edwards-Anderson order parameter. Below we will show numerical evidence that for small and moderate $\lambda, T_{g}$ is nonzero. In the low temperature state there is no long range ferromagnetic order so the system is certainly not a ferromagnet; we will choose to call the frozen state a "spin glass," although random systems with short range ferromagnetic or antiferromagnetic order are often referred to as "cluster glasses" in an experimental context.

One of the established numerical techniques for determining the ordering temperature in ferromagnets and spin glasses is through finite size scaling using the Binder cu- mulant [4]. For sets of samples of different sizes $L$, the fluctuations of the autocorrelation function $q(t)$ in equilibrium are recorded. The Binder cumulant at temperature $T$ is defined as

$$
g_{L}(T)=\frac{1}{2}\left(3-\frac{\left\langle q^{4}\right\rangle}{\left\langle q^{2}\right\rangle^{2}}\right)
$$

and is dimensionless; for sets of samples with fixed $L$ values, the series of curves $g_{L}(T)$ as a function of temperature intersect at the ordering temperature. We have carried out simulations using heat bath dynamics and sequential spin by spin updates on samples up to $L=12$. Five hundred samples were used at each size for each value of $\lambda$, and standard precautions were taken to assure that thermal equilibrium was achieved [4]. We show in Fig. 1 the $g_{L}$ data for three values of $\lambda$, and it can be seen that for the smallest value there is a clear intersection point. For $\lambda=0.7$ the $g_{L}$ curves come together without any clear fanning out at lower temperatures, but we consider that the meeting point represents the freezing temperature here also. For $\lambda=1$ it is hard to say if the curves will intersect above $T=0$. (Very similar behavior with almost unobservable fanning out below $T_{g}$ is seen in 3D ISGs $[2,4]$.) The behavior of the freezing temperature as a function of $\lambda$ is shown in the inset of Fig. 2; at small $\lambda, T_{g}$ extrapolates back to the Onsager $T_{c}$, and, with increasing $\lambda, T_{g}$ drops as might be expected, because for high enough $\lambda$ we will recover the 2D random interaction near neighbor ISG with its zero temperature ordering. It appears that the limiting value of $\lambda$ for nonzero $T_{g}$ is in the region of 1.0.

In conventional second order transitions, $g_{L}\left(T_{c}\right)$ has a universal value for all systems in a given class; for instance, for the 2D Ising ferromagnet the magnetism Binder parameter $g_{L m}\left(T_{c}\right)=0.91603$ [5], and we find the autocorrelation Binder parameter $g_{L}\left(T_{c}\right)=0.42$. We can note in Fig. 1 that $g_{L}\left(T_{g}\right)$ changes with $\lambda$, so, for the present systems of 2D ISGs, universality is not obeyed. For the range of $\lambda$ that we have studied, $g_{L}\left(T_{g}\right)$ appears to be tending regularly to a value near 1 as $T_{g}$ is tending to zero. At still higher $\lambda$ values, $g_{L}(0)$ will presumably tend to 0.83 , which is the standard $2 \mathrm{D} \pm J$ ISG zero temperature value $[2,4]$. We can 
(a)

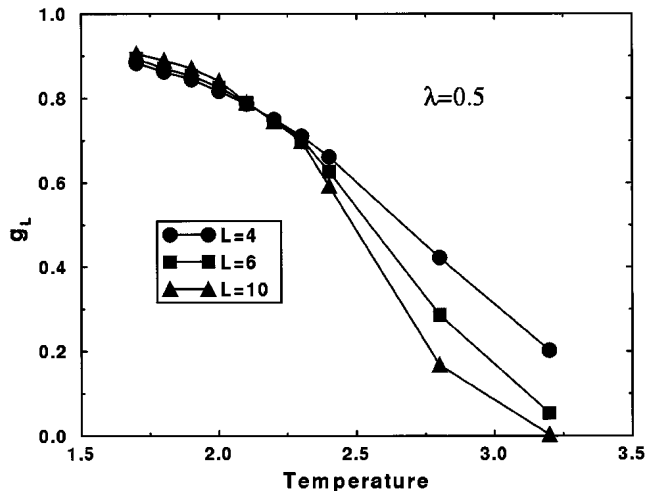

(b)

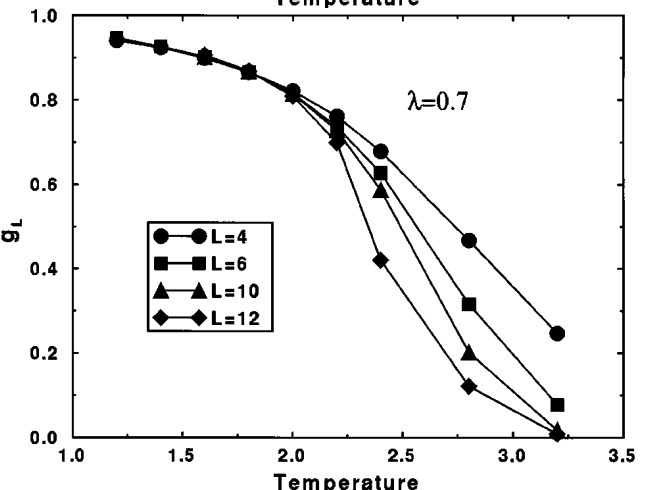

(c)

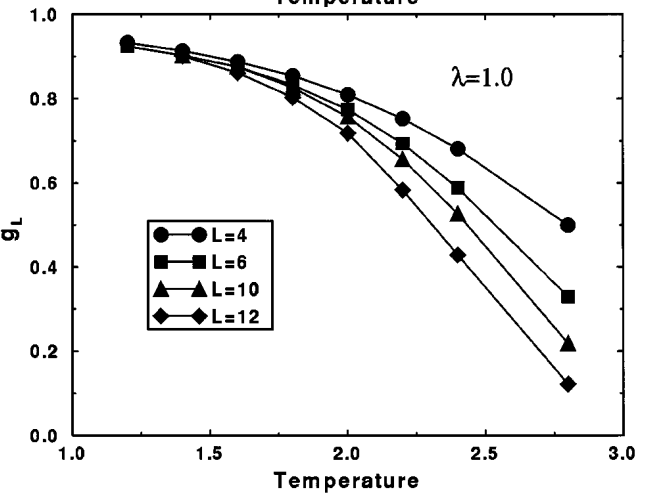

FIG. 1. The measured Binder cumulants as a function of size $L$ and temperature $T$ for $\lambda=0.5,0.7$, and 1.0, respectively.

note that a closely related nonrandom 2D Ising system, having first and second neighbor interactions with regular frustration, shows nonuniversality of critical exponents when a control parameter representing the ratio of the two interaction strengths is varied [6]. There is also strong evidence for nonuniversality of the critical exponents in canonical ISGs at dimension 3 and above [7]. We have not yet estimated the values of the critical exponents for the present family of 2D ISGs.

Once the existence of finite ordering temperatures is established, we can carry out simulations to measure the standard physical properties for these 2D ISGs in order to compare with those of other ISGs. In Fig. 2 we show the specific heats for the same three values of $\lambda$. It can be seen that the specific heats show broad maxima at temperatures higher than the respective ordering temperatures,

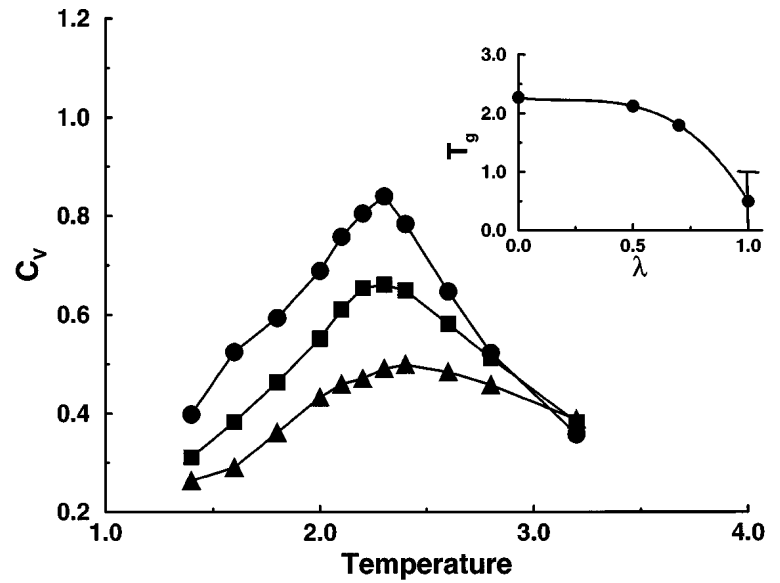

FIG. 2. The specific heat averaged over samples with $L=40$ for $\lambda=0.5,0.7$, and 1.0 (circles, squares, and triangles, respectively). Inset: $T_{g}$ as a function of $\lambda$.

and smooth featureless behavior through the freezing transition. This is precisely what is observed for standard ISGs in dimension 3 [8], 4, and 5 [9], and appears to be a universal characteristic of the ISG transition below the upper critical dimension. For the present systems the maximum of the specific heat corresponds to a saturation of the ferromagnetic correlation length in each sublattice as the sample is cooled; the ordering process can be thought of as consisting of, first, the formation of finite size ferromagnetic domains, and then of a freezing at a lower temperature where the domain movements are hindered by the random near neighbor interactions.

Another parameter accessible from simulations is the damage spreading [10]. Damage spreading is measured numerically by using heat bath dynamics and applying the same random update parameter at each update step to two replicas of the same system; $D(T)$ is defined as the normalized Hamming distance between the two replicas (i.e., the fraction of spins having opposite orientations) at long times when this protocol is applied. While ferromagnets have $D(T)=0$ down to $T_{c}$, standard ISGs have been found to show nonzero $D(T)$ until temperatures well above $T_{g}[10,11]$; it has been pointed out that for ISGs in dimensions 3 and 4 , as the temperature is reduced, $D(T)$ tends to $1 / 2$ at a temperature very close to $T_{g}$ [11]. We have studied damage spreading in the present 2D ISGs. Two random initial replicas of each 2D system were chosen; these replicas were annealed independently to thermal equilibrium at each temperature, and then the damage spreading procedure was applied for long times. The results, Fig. 3, show that the damage spreading temperature $T_{D}$ where $D(T)$ first becomes nonzero is much higher than $T_{g}$, and that in each case on cooling $D(T)$ tends to $1 / 2$. For $\lambda=0.5$ this temperature is indistinguishable from the $T_{g}$ we have estimated from the Binder cumulant data. $D(T)$ then remains equal to $1 / 2$ for 


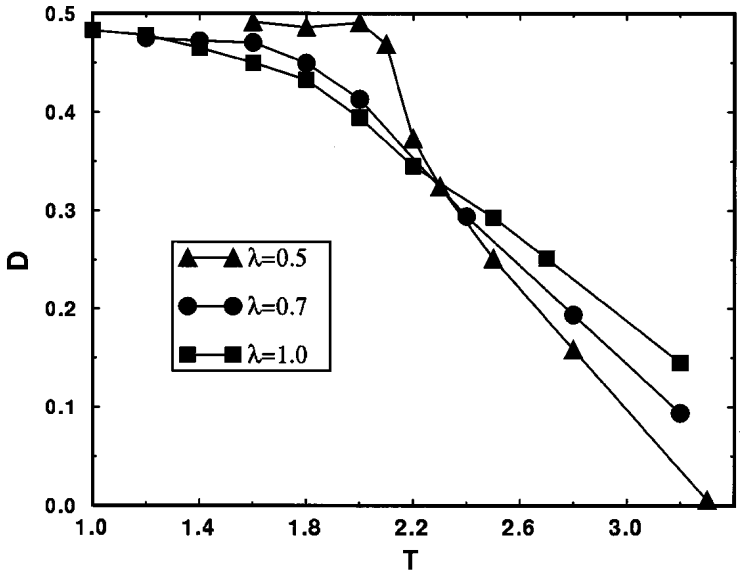

FIG. 3. The damage spreading parameter $D(T)$ as a function of temperature (see text) for $\lambda=0.5$ (triangles), 0.7 (circles), and 1.0 (squares) for $40 \times 40$ spins.

lower temperatures. The behavior is similar but less clear cut for the other two values of $\lambda$ (the lowest temperature points may be slightly low because of the very long times needed to achieve complete thermal equilibrium). The general behavior of the damage spreading parameter appears to be the same in these 2D ISGs as in ISGs in dimensions 3 and 4 . The use of the empirical criterion $D\left(T_{g}\right)=1 / 2$ gives a convenient method to check $T_{g}$ values.

An advantage of the present systems over conventional ISGs is that "snapshots" of the instantaneous configurations of the spins are informative because we are in dimension 2. We show in Fig. 4 two snapshots of the same $\lambda=0.5$ system at temperature $T=1.5$, well below $T_{g}$. The images were produced by slowly cooling two independent random replicas of the same system by successive steps to the final temperature. As expected, there are four types of domains: If we call one sublattice $A$ and the other $B$, then the domains are $A$ up $B$ up (white), $A$ up $B$ down (white/black checkerboard), $A$ down $B$ up (black/white), and $A$ down $B$ down (black). Here the domain sizes are typically 25 by 25 spins. (The simulations confirm that the typical domain size below $T_{g}$ gets smaller as $\lambda$ is increased.) For this sample at this temperature, the two configurations are almost frozen; averaging over a further 10000 Monte Carlo steps per spin (MCS) only blurs the domain frontiers somewhat. To a good approximation, the images then represent two Gibbs states of the system. By inspection it can be seen that the two states are neither quasi-identical, nor are they quasimirror images of each other with all the spins reversed. The detailed structures of the two states are quite different, suggesting that there is an infinity (or at least a large number) of alternative Gibbs states for this ISG. This conclusion appears to be incompatible with the two ground state images which have been proposed heuristically for ISGs [12], but the (a)

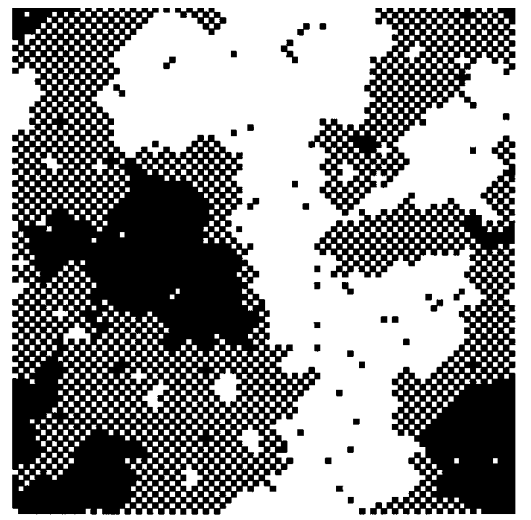

(b)

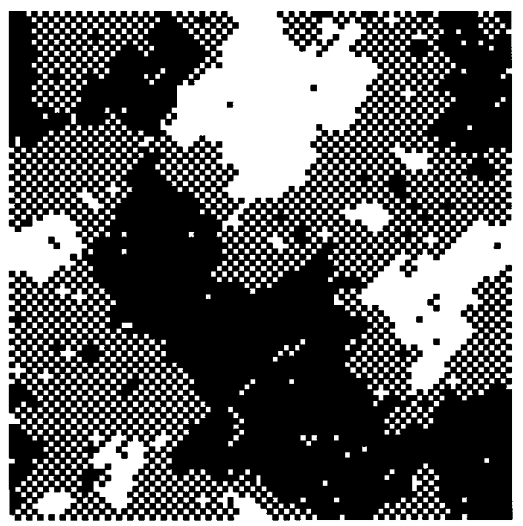

(c)

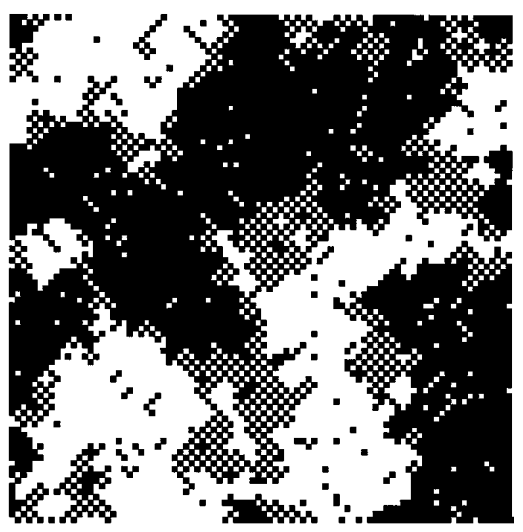

FIG. 4. Snapshots of two replicas $A, B$ of the same $100 \times$ 100 spin system with $\lambda=0.5$ at $T=1.5$ after 10000 MCS anneal. The two replicas were cooled independently from two initially randomly chosen configurations. On each site, black is up and white is down. The third frame is the difference between the two configurations $A$ and $B$ : black, $S_{i}^{A}=S_{i}^{B}$; white, $S_{i}^{A}=-S_{i}^{B}$.

phase space could resemble that of the Parisi solution of the infinite range SK model [13].

In conclusion, we have demonstrated that 2D ISG systems with nonzero freezing temperatures exist. We have shown data for a certain number of parameters for a new family of 2D ISGs, where we have found that the observed behavior is very similar to that seen in simulations on standard ISGs in dimension 3. A more comprehensive study of the 2D ISGs and a detailed comparison with results from 
higher dimensions should establish what properties are always necessarily associated with spin glass ordering and should help understanding of the spin glass phenomenon in general. As far as experimental comparisons are concerned, it might be worth reconsidering the data on 2D ISG materials without making the a priori assumption that the ordering temperature is necessarily zero.

Finally, the general philosophy behind the present study may help to resolve a long standing paradox. Real threedimensional spin glass materials where the spins can be considered to be basically Heisenberg show well-defined nonzero freezing temperatures; on the other hand, in numerical work and on theoretical grounds it appears that Heisenberg spins with random near neighbor interactions should not show a freezing transition at a finite temperature [14] (but it should be noted that, with vector spins, chirality should also play a role [15], and that a simulation of a Heisenberg spin glass with RKKY interactions shows a finite $T_{g}[16]$ ). Now we have just seen that, if we have a set of random near neighbor interactions only, a 2D Ising system has no finite temperature ordering, but the system can be a spin glass with a finite $T_{g}$ if there are both random and nonrandom interactions. Perhaps the same rule applies to 3D Heisenberg systems. It may well be that all real life Heisenberg spin glasses have both random and nonrandom interactions (and so, in a sense, might be classified as cluster glasses [17]). Thus even in the canonical CuMn alloys the short range magnetic ordering which has been observed with neutrons [18] may be essential for the spin glass transition to occur at a finite temperature.

We would like to thank Lorenzo Bernardi for his contribution for programming. The numerical calculations were carried out thanks to a time allocation provided by the Institut du Developpement et des Ressources en Informatique Scientifique (IDRIS). N. L. is supported by a CNPq scholarship.
*Electronic address: lemke@lps.u-psud.fr

${ }^{\dagger}$ Permanent address: Instituto de Física, Universidade Federal do Rio Grande do Sul, C.P. 15051, 91501-970, Porto Alegre, RS, Brazil.

*Electronic address: campbell@1ps.u-psud.fr

[1] K. Binder and A. P. Young, Rev. Mod. Phys. 58, 801 (1986).

[2] R. N. Bhatt and A.P. Young, Heidelberg Colloquium on Glassy Dynamics, edited by J.L. van Hemmen and I. Morgenstern (Springer-Verlag, Berlin, 1987).

[3] Y. Imry and S. K. Ma, Phys. Rev. Lett. 35, 1399 (1975).

[4] R. N. Bhatt and A.P. Young, Phys. Rev. B 37, 5606 (1988).

[5] K. Binder, Z. Phys. B 43, 119 (1981); G. Kamieniarz and H. W. J. Blöte, J. Phys. A 26, 201 (1993).

[6] K. Minami and M. Suzuki, Physica (Amsterdam) 195A, 457 (1993).

[7] L. Bernardi and I. A. Campbell, Phys. Rev. B 52, 12501 (1995).

[8] A. T. Ogielski, Phys. Rev. B 32, 7384 (1985).

[9] L. Bernardi and I. A. Campbell (unpublished).

[10] B. Derrida and G. Weisbuch, Europhys. Lett. 4, 657 (1987); B. Derrida, Phys. Rep. 184, 207 (1989).

[11] I. A. Campbell, Europhys. Lett. 21, 959 (1993).

[12] D. S. Fisher and D. A. Huse, Phys. Rev. Lett. 56, 1601 (1986).

[13] G. Parisi, Phys. Rev. Lett. 50, 1946 (1983); E. Marinari, G. Parisi, J. Ruiz-Lorenzo, and F. Ritort, Phys. Rev. Lett. 76, 843 (1996).

[14] J. A. Olive, A. P. Young, and D. Sherrington, Phys. Rev. B 34, 6341 (1986); H. Yoshino and H. Takayama, Europhys. Lett. 22, 631 (1993).

[15] H. Kawamura, J. Phys. Soc. Jpn. 64, 26 (1995).

[16] F. Matsubara and M. Iguchi, Phys. Rev. Lett. 68, 3781 (1992).

[17] Ordering in cluster glasses is discussed by Th. M. Nieuwenhuizen and C. N. A. van Duin (unpublished).

[18] J. W. Cable, S. A. Werner, G. P. Felcher, and N. Wakabayashi, Phys. Rev. B 29, 1268 (1984). 